\title{
AN INTERPOLATION THEOREM FOR ADJOINT FUNCTORS
}

\author{
S. A. HUQ
}

0. Introduction. In this paper we present a category theoretic generalization of the construction of the tensor algebra or symmetric algebra of a module which proceeds by representing the module as the quotient of the free module on the underlying set of the given module by its module of relations, then obtaining the tensor algebra as the quotient of the free algebra on the underlying set of the module modulo, the ideal generated by the module of relations, thus giving a uniform construction of many universal gadgets used in algebra.

We use the notation of MacLane [1].

1. Definition. Let $\mathcal{C}$ and $B$ be two categories and $\Phi: \mathcal{C} \rightarrow ß$ a functor. (We shall explicitly work in the case when the functor is covariant, dualization will be left for the reader.) Then for an object $B \in \mathbb{B}$, a universal arrow from $B$ to $\Phi$ is a pair $\langle C, \gamma\rangle$ with $C$ an object of $\mathrm{e}$ and $\gamma: B \rightarrow \Phi(C) \in \Theta$, such that to every pair $\left\langle C^{\prime}, \mu\right\rangle$ with $C^{\prime} \in \mathcal{C}$ and $\mu: B \rightarrow \Phi\left(C^{\prime}\right)$, there is a unique arrow $\nu: C \rightarrow C^{\prime}$, with $\Phi(\nu) \gamma=\mu$. Equivalently $\langle C, \gamma\rangle$ is universal from $B$ to $\Phi$ when the pair $\langle C, \gamma\rangle$ is an initial object in the comma category $B / \Phi$, whose objects are the arrows $B \rightarrow \Phi\left(C^{\prime}\right)$, for $C^{\prime} \in \mathcal{C}$.

2. Main theorem. Suppose $\Psi: \beta \rightarrow Q$ and $\Phi: \mathcal{C} \rightarrow B$ are covariant functors and $A \in Q$. Then if $B_{1} \in \beta$ and $\Psi\left(B_{1}\right)$ has a universal arrow to $\Psi$ and to $\Psi \Phi$, then under the hypotheses:

(a) If $\Psi(\beta)$ is a retraction in $\alpha$, then there exist $\beta_{1}, \beta_{2}$ in $B$ so that $\beta$ is the coequalizer of $\beta_{1}$ and $\beta_{2}$, and

(b) The class $\left\{\gamma \in \mathbb{C} \mid \Phi(\gamma) \beta_{1}=\Phi(\gamma) \beta_{2}\right\}$ consists of all morphisms of the form $\gamma^{\prime} \rho$ for some epimorphism $\rho$,

there exists a universal arrow from $B_{1}$ to $\Phi$.

Proof. Let $\langle C, \mu\rangle$ and $\langle B, \nu\rangle$ be universal from $\Psi\left(B_{1}\right)$ to $\Psi \Phi$ and $\Psi$ respectively: since $\langle B, \nu\rangle$ is universal from $\Psi\left(B_{1}\right)$ the morphism $\mu: \Psi\left(B_{1}\right) \rightarrow \Psi \Phi(C)$ determines a unique morphism $\mu_{1}: B \rightarrow \Phi(C)$, such that

$$
\Psi\left(\mu_{1}\right) \nu=\mu .
$$

Also the identity morphism of $\Psi\left(B_{1}\right)$ determines a unique $\delta$ such that

Received by the editors February 28, 1969 and, in revised form, August 28, 1969. 


$$
\Psi(\delta) \nu=1_{\Psi\left(B_{1}\right)} .
$$

We shall use our hypothesis (a) only for this $\delta$, and (b) only for $\beta_{1}=\mu_{1} \delta_{1}$ and $\beta_{2}=\mu_{1} \delta_{2}$, where $\delta$ determines the pair $\delta_{1}, \delta_{2}$ under hypothesis (a).

Thus, since $\Psi(\delta)$ is a retraction, there exists a parallel pair

$$
\underset{\delta_{2}}{\stackrel{\delta_{1}}{\longrightarrow}}
$$

such that $\delta=$ coequalizer of $\delta_{1}$ and $\delta_{2}$ (hypothesis (a)). Let us choose the fixed $\rho$ to be a mapping $C \rightarrow C_{1} \in \mathcal{C}$ such that $\Phi(\rho) \beta_{1}=\Phi(\rho) \beta_{2}$.

Since $\delta=$ coequalizer $\left(\delta_{1}, \delta_{2}\right)$, there exists a $\omega: B_{1} \rightarrow \Phi\left(C_{1}\right)$ satisfying

$$
\omega \delta=\Phi(\rho) \mu_{1} \text {. }
$$

We shall check that $\left(C_{1}, \omega\right)$ is a universal arrow from $B_{1}$ to $\Phi$. So let $C^{*} \in \mathcal{C}$ and let $\theta: B_{1} \rightarrow \Phi\left(C^{*}\right)$ be an arrow in $B$. Since $\langle C, \mu\rangle$ is universal from $\Psi\left(B_{1}\right)$, the morphism $\Psi(\theta): \Psi\left(B_{1}\right) \rightarrow \Psi \Phi\left(C^{*}\right)$ determines a unique $\kappa: C \rightarrow C^{*}$, such that

$$
\Psi \Phi(\kappa) \mu=\Psi(\theta) .
$$

Now $\Psi(\theta)=\Psi(\theta) 1_{\Psi\left(B_{1}\right)}=\Psi(\theta) \Psi(\delta) \nu=\Psi(\theta \delta) \nu$.

Also $\Psi \Phi(\kappa) \Psi\left(\mu_{1}\right) \nu=\Psi(\theta)=\Psi(\theta \delta) \nu$, i.e. $\Psi\left(\Phi(\kappa) \mu_{1}\right) \nu=\Psi(\theta \delta) \nu$. As $\langle B, \nu\rangle$ is a universal arrow from $\Psi\left(B_{1}\right)$ to $\Psi$, we must have

$$
\Phi(\kappa) \mu_{1}=\theta \delta \text {. }
$$

Now $\Phi(\kappa) \beta_{1}=\Phi(\kappa) \mu_{1} \delta_{1}=\theta \delta \delta_{1}=\theta \delta \delta_{2}=\Phi(\kappa) \beta_{2}$, hence by hypothesis (b) there exists a $\lambda: C_{1} \rightarrow C^{*}$, such that

$$
\kappa=\lambda \rho .
$$

Again

$$
\begin{aligned}
\Psi \Phi(\lambda) \Psi(\omega) \Psi(\delta) \nu & =\Psi \Phi(\lambda) \Psi(\omega \delta) \nu \\
& =\Psi \Phi(\lambda) \Psi\left(\Phi(\rho) \mu_{1}\right) \nu \quad \text { from (2) } \\
& =\Psi \Phi(\lambda \rho) \Psi\left(\mu_{1}\right) \nu \\
& =\Psi \Phi(\kappa) \mu=\Psi(\theta) \quad \text { from (1), (5) and (3) } \\
& =\Psi(\theta \delta) \nu .
\end{aligned}
$$

Now since $(B, \nu)$ is universal, we immediately have

$$
\theta \delta=\Phi(\lambda) \omega \delta
$$

which in turn gives

$$
\theta=\Phi(\lambda) \omega
$$


since $\delta$ is an epimorphism. Also using the fact that $\rho$ is an epimorphism, one can check that $\lambda$ is unique satisfying (6).

Next if for each object $B \in B$, there is a universal arrow $\left(t(B), \gamma_{B}\right)$ from $B$ to $\Phi\left(\gamma_{B}\right.$ being the arrow $B \rightarrow \Phi t(B)$ in $\left.B\right)$ then the function $t$ can be considered as a functor and we have the

Interpolation Theorem. Suppose $\stackrel{\mathrm{e}}{\stackrel{\Phi}{\rightarrow}} \leftrightarrow \stackrel{\Psi}{\rightarrow} Q$ is a diagram of categories and functors with $\Phi^{\prime}$ and $\Psi^{\prime}$ as coadjoints for $\Psi$ and $\Psi \Phi$, respectively, then we have a coadjoint for $\Phi$ whenever hypotheses (a) and (b) of the main theorem hold.

3. Application. Our theorem essentially reduces Lawvere's famous theorem [2] "Every algebraic functor has an adjoint" to the existence of free algebras over sets, i.e. the existence of an adjoint to the forgetful functor guarantees the existence of an adjoint for all algebraic functors.

More clearly let $\mathscr{D}$ be a small category and $\mathcal{G}$ an arbitrary one. We consider the functor category $\mathcal{G}^{D}$ and the imbedding $\mathcal{G} \rightarrow \mathcal{G}^{D}$ which carries any $G \in \mathrm{Obj} g$ into the obvious constant functor. Now if $\mathcal{G}$ is algebraic we have the commutative diagram

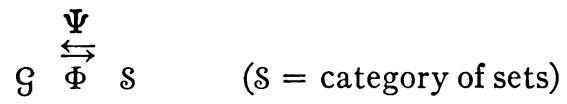

$$
\begin{aligned}
& \left.\mathcal{G}^{\mathcal{D}} \underset{\Phi^{D}}{\stackrel{\Psi^{D}}{\leftrightarrows}} S^{\mathbb{D}} \quad \text { ( } \Phi=\text { forgetful functor }\right)
\end{aligned}
$$

of adjoint pairs and the fact that every algebraic category has direct limits (4) follows again from the fact that the forgetful functor $\Phi$ has an adjoint $\Psi$, by interpolating in the diagram

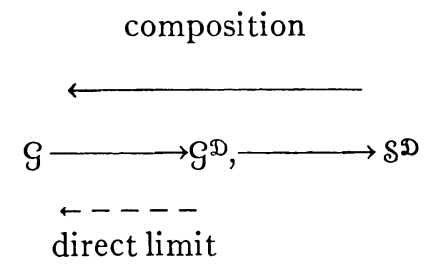

By similar methods one obtains results as in Frei [3, Satz 2.9].

4. Acknowledgements. The author acknowledges excellent criticism from the referee which contributed to the improvement of this paper. Original version of this was written by the author in 1962 under Fröhlich's supervision. 


\section{REFERENCES}

1. S. Mac Lane, Categorical algebra, Bowdoin Summer Seminar, 1969.

2. F. W. Lawvere, Functorial semantics of algebraic theories, Thesis, Columbia University, New York, 1963.

3. A. Frei, Freie Objekte und multiplikative Strukturen, Math. Z. 93 (1966), 109141. MR 33 \#5696.

4. G. Georgescu and D. Popescu, On algebraic categories, Rev. Roumaine Math Pures Appl. 13 (1968), 337-342. MR 38 \#1140.

Université de Montréal, Montréal, Canada 